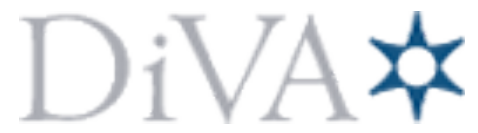

http://kth.diva-portal.org

This is an author produced version of a paper published in the Journal on Multimodal User Interfaces.

This paper has been peer-reviewed but does not include the final publisher proof-corrections or journal pagination.

Citation for the published paper:

Gaël Dubus

"Evaluation of four models for the sonification of elite rowing"

Journal on Multimodal User Interfaces, Special issue on Interactive Sonification, in press, published online 26 January 2012

URL: http://dx.doi.org/10.1007/s12193-011-0085-1

Access to the published version may require subscription.

Publish with permission from: Springer 


\title{
Evaluation of four models for the sonification of elite rowing
}

\author{
Gaël Dubus
}

Received: date / Accepted: date

\begin{abstract}
Many aspects of sonification represent potential benefits for the practice of sports. Taking advantage of the characteristics of auditory perception, interactive sonification offers promising opportunities for enhancing the training of athletes. The efficient learning and memorizing abilities pertaining to the sense of hearing, together with the strong coupling between auditory and sensorimotor systems, make the use of sound a natural field of investigation in quest of efficiency optimization in individual sports at a high level. This study presents an application of sonification to elite rowing, introducing and evaluating four sonification models. The rapid development of mobile technology capable of efficiently handling numerical information offers new possibilities for interactive auditory display. Thus, these models have been developed under the specific constraints of a mobile platform, from data acquisition to the generation of a meaningful sound feedback. In order to evaluate the models, two listening experiments have then been carried out with elite rowers. Results show a good ability of the participants to efficiently extract basic characteristics of the sonified data, even in a non-interactive context. Qualitative assessment of the models highlights the need for a balance between function and esthetics in interactive
\end{abstract}

This work was supported by the Swedish Research Council, Grant Nr. 2010-4654, by the Olympic Performance Center (OPC) SONEA project, and by the EU-ICT SAME project (FP7-ICT- STREP-215749) http://www.sameproject.eu.

Gaël Dubus

KTH Royal Institute of Technology,

School of Computer Science and Communication,

Department of Speech, Music and Hearing

Lindstedtsvägen 24, 10044 Stockholm, Sweden

Tel. : $+46-87907857$

Fax : $+46-87907854$

E-mail: dubus@kth.se sonification design. Consequently, particular attention on usability is required for future displays to become widespread.

Keywords Sonification · Rowing - Sculler · Sports · Accelerometer

\section{Introduction}

1.1 Interactive sonification in sports: an overview

Sonification is a relatively recent field of research, yet it already offers many possibilities for practical application. Characterized by real-time interaction between a subject and an auditory display, interactive sonification is particularly suitable for sport context. Many examples exploiting the use of athlete body motion as the main input data stream have been introduced during the past few years. Taking advantage from the strong learning and memorizing abilities associated with the sense of hearing, the perception of complex sport movements can be enhanced by additional auditory information as shown by Effenberg [3]. The multiple advantages of auditory feedback in sports motivated research that led to successful experiments and innovations in several domains.

Following the concept of sport activities specially adapted for visually impaired athletes such as torball and blind football, the framework AcouMotion [13] enables the design of new sports games like Blindminton, an adapted version of badminton making use of various sonification techniques to "create a new channel of proprioception". Sonification methods for physical rehabilitation have also appeared. A convincing example is given by Godbout [7], who used interactive sonifica- 
tion in a successful way for rehabilitating a speed skater suffering from Lost Move Syndrome.

Optimization of performance is certainly the main objective in sports technology research though. Approaching optimal efficiency in individual sports is a major concern for athletes and trainers, especially at a high level as differences in performances tend to become smaller. Biomechanical studies account for the most significant part of research towards an optimal technique, by identifying the influence that specific kinetic quantities (forces, momentums) can have on the resulting motion patterns, as well as by studying kinematic quantities characterizing the motion itself. These studies provide tools for estimation of power production and therefore openings for efficiency optimization of the performance. In contrast, few investigations have been conducted on the possibility to influence the training methods of the athlete, for example by enhancing the perception of his own movements in order to take advantage of the quick development of the processes involved in embodied cognition. These processes are associated to sensory feedback mainly consisting of haptic, visual, and auditory information. Modifying the haptic feedback would be both technically challenging and potentially obtrusive to the athlete. On the other hand, visual and auditory enhancement of the training can easily be effective without overloading the cognitive system. Sonification frameworks aiming at improving the self-perception of one's body movements have recently been developed for this purpose, such as MotionLab Sonify [3], Physiosonic [30] and AcouMotion, which can also be used in this context. The universal character of sonification implies that a large number of sports can potentially benefit from this strategy, which has already been applied among others to golf [17], aerobics [14], running [4] and German wheel [16].

This article presents four interactive sonification designs aiming at influencing training in single sculler rowing. Instead of only sonifying the movements of the rower's body, we consider the boat and the oars as an extension of this body. In this way, the enhanced proprioception is meant to apply to this extended body. The aim of the project is that the athlete will learn to row using the sonification system as he would learn how to play a musical instrument. The present experiment is however limited to the design and evaluation of sonification models using a limited amount of pre-recorded motion data.

\subsection{Previous work in sonification of rowing}

Sonification of rowing has recently been tackled in a project by researchers from the University of Hamburg.
Since 2008, Schaffert et al. have been focusing on the influence of using a real-time auditory display on the rowing cycle, mainly through the analysis of the structure of acceleration time series of the boat. In a series of publications [21,23-25], the authors were concerned with explaining the cognitive mechanisms involved in the perception-action loop. By contrast, less attention was given to the esthetic qualities of the auditory display, and little information is available about the sound material used in the sonification. An evaluation of the system was performed through surveys answered by athletes and coaches, showing their good acceptance of the principle of sonification. Probably constrained by the experimental protocol of on-water testing, the nature of these questionnaires (polar questions and free comments) did not enable advanced quantitative evaluation and the focus was therefore set on the efficiency. Promising results were presented, showing a significant improvement of the average velocity, which was explained by a better synchronization between the rowers in a crew, and to a certain extent by an amelioration in individual technique, since there was likewise an improvement for a single sculler.

Esthetics of the sonification was first considered in [22]: in addition to the original sonification - a pure tone with gliding frequency called sinification, six advanced models were designed during a workshop gathering researchers in sound interaction. A large palette of sonic material was exploited, such as instrument sounds, environmental rowing sounds, ecological metaphors, artificially generated techno music and vocal formants. Similarities and differences between some of these models and the ones introduced in the present article are discussed in Section 3.2.4. The models were designed using acceleration of a rowing boat as input data during the workshop, but no evaluation was conducted on the differences between the models concerning information extraction and esthetic preferences of the athletes in the context of elite rowing. Barrass et al. [1] further extended this work by designing SweatSonics, a technology probe specifically designed for interactive sonification of recreational sporting activities, implementing the models realized in [22]. The fact that no specific task was assigned to the subjects shows that functional evaluation was left aside to focus exclusively on esthetic aspects. By recording activity and choices with respect to sonification models, the authors could observe evolutions in the behavior of the users and identify their favorite model. Interviews were subsequently realized in order to compare the opinion of the subjects with the analysis of their own log data, which turned out to be consistent with each other. The results of this study reveal the existence of general trends in the subjects' 
preferences, but also a great diversity in the ranking of the models, which underlines differences in personal requirements concerning esthetics of an auditory display.

A robust evaluation phase is often neglected by sonification designers. In many cases, the evaluation is reduced to a binary result: either the auditory display allows to perform the task it was designed for, or it doesn't. Due to the proximity between the topics, it is interesting to compare our results with conclusions by Schaffert, Barrass, et al. [1,21-25]. Nevertheless, the setting of our experiment is different from those in the two series of publications mentioned above: in the first series, only one simple sonification model was tested with elite rowers and evaluated from a functional perspective. In the second series, several sound models were assessed but the experiment was conducted in a different context, namely a broad range of outdoor activities instead of exclusively rowing. In addition, the subjects were researchers in Human-Computer Interaction (HCI), which could possibly lead to different results when testing with elite athletes, especially since the evaluation was concentrated on esthetic aspects of the sonification. The objective of the present work is to perform a quantitative evaluation based on listening tests carried out by elite and casual rowers, taking into account both esthetic and functional aspects.

\section{Biomechanics of rowing}

Numerous biomechanical studies of rowing have been carried out since the end of the nineteenth century and presenting a comprehensive review of the existing literature goes beyond the objectives of this article. Nevertheless, since the properties of the input data are of primary importance in any sonification work, an overview of previous work describing kinematic and kinetic quantities involved in rowing is presented here. Kleshnev [18] tackles this question from a pragmatic perspective by investigating the different types of sensors allowing to perform measurements in a rowing boat. In this way, he sets up a list of measurable quantities which can be considered as available for analysis. This list includes kinematic quantities related to the boat, to the oars, to the sliding seats and to the athlete himself: acceleration, velocity, position, angles, three-dimensional orientation (yaw, pitch, roll), position of the trunk. Kinetic quantities are also listed: oar force - the main factor of propulsion, and forces measured at several places of the boat: foot-stretchers, oarlocks, gates and handles. Various types of sensors (potentiometers, accelerometers, impellers, gauges) can be associated to these biomechanical variables. Environmental parameters such as wind speed and direction, and water temperature round out the set of measurable parameters.

Based on the analysis of some of these parameters, McBride [19] and Soper and Hume [26] provide guidelines to optimize the rowing cycle. McBride starts from the dissection of a rowing stroke (catch, drive phase, release, recovery phase) to discuss the influence of diverse biomechanical variables on dynamic features of the rowing cycle, in particular those related to the propulsion: oar motion, blade forces, boat velocity. Optimization of efficiency is tackled through the study of force-angle closed curves, the area under which represents the total work produced during a stroke cycle. The author elaborates on the means to achieve a more efficient shape of the curve - for example with an "explosive leg drive at the catch" - and states that an optimal curve would be different depending on the position of the rower in the case of non-single scull boats. Both studies agree on the fact that excessive variations in the boat velocity induce a detrimental energy dissipation due to friction with water. However, since many other parameters should be taken into account, a minimal value for this dissipation (in theory occuring for a constant velocity) would not give an optimal cycle yet. This is pointed out by Hofmijster in his doctoral dissertation [15] by arguing that sliding seats induce more variations in boat velocity but allow for more power production than fixed seats. With a particular focus on the different causes of energy loss, he tries to determine the influence of stroke rate on rowing efficiency. As in the aforementioned studies, energy dissipation due to velocity fluctuations is said to have a significant effect on efficiency, as well as dissipation caused by the blades while pushing the water. No significant effect on gross efficiency could be observed for energy losses due to the back-andforth movement of the rower's body, depending itself on the stroke rate. Finally, the author demonstrates how rowing skills, being characterized by a better coordination of timing between various kinetic quantities, can affect power loss due to velocity fluctuations and therefore improve rowing efficiency.

Research progress in rowing biomechanics provide useful hints for efficiency optimization, yet further investigation would be required to be able to define a "perfect" rowing cycle as given for example by a standard model curve. If such a model was available, it would be possible to design auditory displays aiming at attracting the rowers towards this reference. At this stage, however, our goal is limited to the improvement of proprioception in order to speed up the progress and to develop analytical skills through interaction with a sonification system. 


\section{Sonification of single scull rowing}

\subsection{Data acquisition}

The aim of this project is to enhance the training process by means of interactive sonification so that it will converge faster and closer to an optimal rowing technique. Whereas there exist various potential uses of a sonification system as for example synchronization between rowers of a crew, we chose to focus on technique improvement for single sculler. The objective to fulfill when looking for the optimal rowing technique is the optimization of the average velocity of the boat that Soper and Hume [26] consider as "the controllable determinant" in a race. Velocity was therefore our main concern and was chosen to be displayed as a continuous auditory feedback in three of our models, whereas acceleration was chosen in the fourth one.

Considering the little space available in a single scull, and with the development of mobile technology, handheld devices represent a natural solution for setting up a sonification system to be used in rowing training. Last generation mobile phones possess the required functionalities to perform the complete process from data acquisition to sound synthesis. Still these devices have limitations with respect to computational resources, and implementing a complete system running efficiently in real-time on a mobile platform represents a real challenge. New types of sensors have also appeared, allowing interactive systems to be more aware of their context of use.

Data were collected on the artificial flatwater course in Račice, Czech Republic, during a training camp with athletes from the Swedish national rowing team. The equipment used for these experiments consisted in a Nokia N95 mobile phone running Symbian S60 operative system and including an accelerometer, a GPS receiver and a MIDI synthesizer, and a couple of wireless Witilt v3.0 triple axis accelerometers from SparkFun Electronics. Thus, only kinematic quantities could be measured. The external accelerometers were preferred to the built-in one, since they had a higher resolution and a wider range $( \pm 6 g)$. The quality of the measurements performed with the GPS receiver turned out to be rather poor, therefore only the acceleration data from the accelerometers were exploited. Threedimensional acceleration data were sent to the mobile phone via a Bluetooth protocol at a frequency of $120 \mathrm{~Hz}$. The complete acquisition process was performed by the phone running a script on the software Python for S60. Finally, a microphone was taped on an outrigger and connected to a MiniDisc recorder placed inside a waterproof storage compartment in order to record the en-

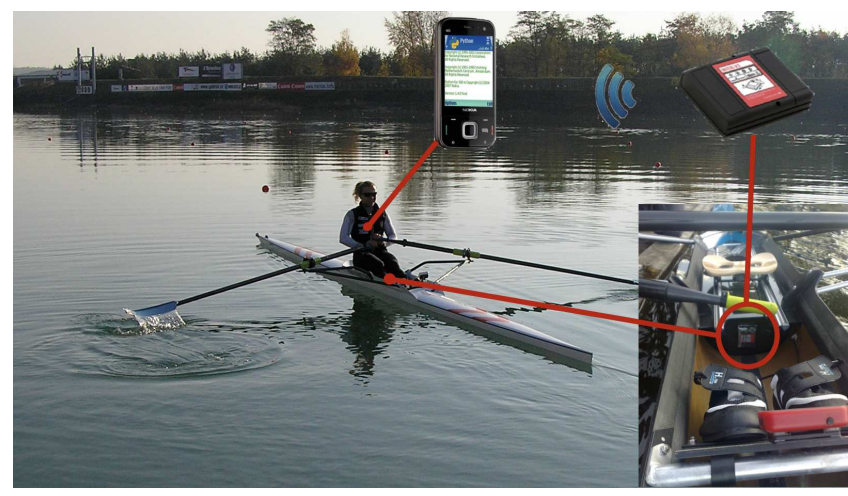

Fig. 1 Equipment: the rower carries a smartphone that can receive GPS and accelerometer data used for the sonification.

vironmental sounds usually heard by the athlete while training.

For the present work, only the direction of propulsion of the boat was taken into account. If values for the velocity were directly integrated from raw acceleration over the complete experiment, they would be totally unrealistic due to the accelerometer drift error. In order to limit this deviation to an offset varying very slowly, the actual data used for the sonification were the difference between this value and a locally averaged velocity computed by a moving average filter. The length of the time window used for the moving average was chosen sufficiently long to correspond to the duration of a few rowing cycles. In this way, the deviation was reduced to the drift error accumulated along the filter window, which was discarded at a later stage of the sonification. A sample of acceleration data is shown in Fig. 2 together with the corresponding integrated velocity. Given that rowers target a specific stroke rate most of the time during their training sessions, it can be useful to compute the instantaneous stroke rate in real-time. For this purpose, a peak detection algorithm was applied to the raw acceleration, as shown in Fig. 3. It is important to note that the only data resulting from physical acquisition are acceleration time series, whereas velocity is computed by the sonification algorithms. In the remainder of the article, input data samples are referred to as acceleration samples such as in Tables 1 and 4 .

Additional sensors could be integrated in future experiments. A GPS receiver of better quality could be used in order to get a better estimate of the absolute value of the boat velocity, which is not required for the present experiment since the sonification models described in Section 3.2.4 taking velocity as input parameter are designed to work with relative velocity fluctuations. Measurements of kinetic quantities would also be valuable in order to develop more advanced soni- 


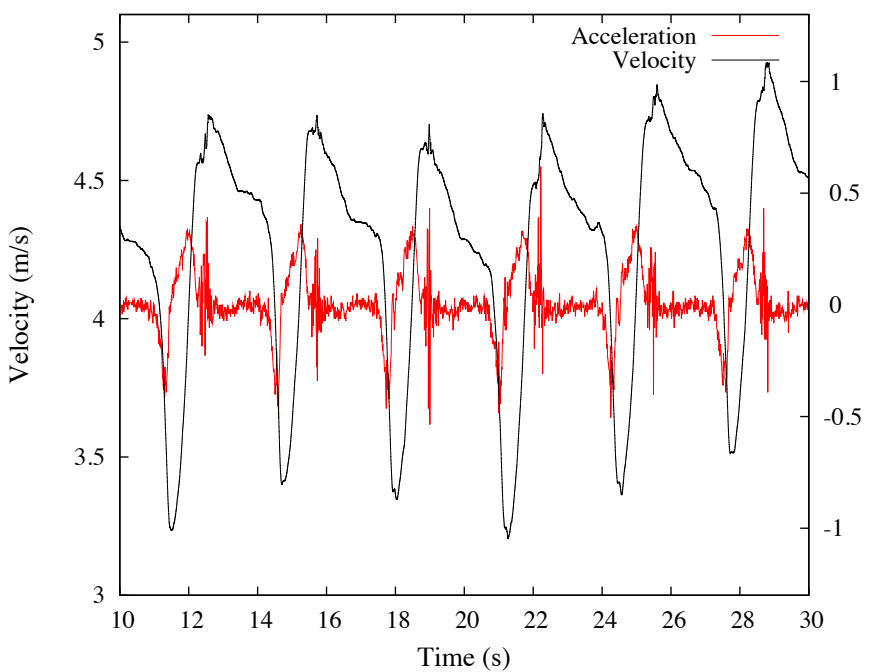

Fig. 2 Acceleration and velocity curves from a training session of a rower from the Swedish national team at 18 strokes per minute.

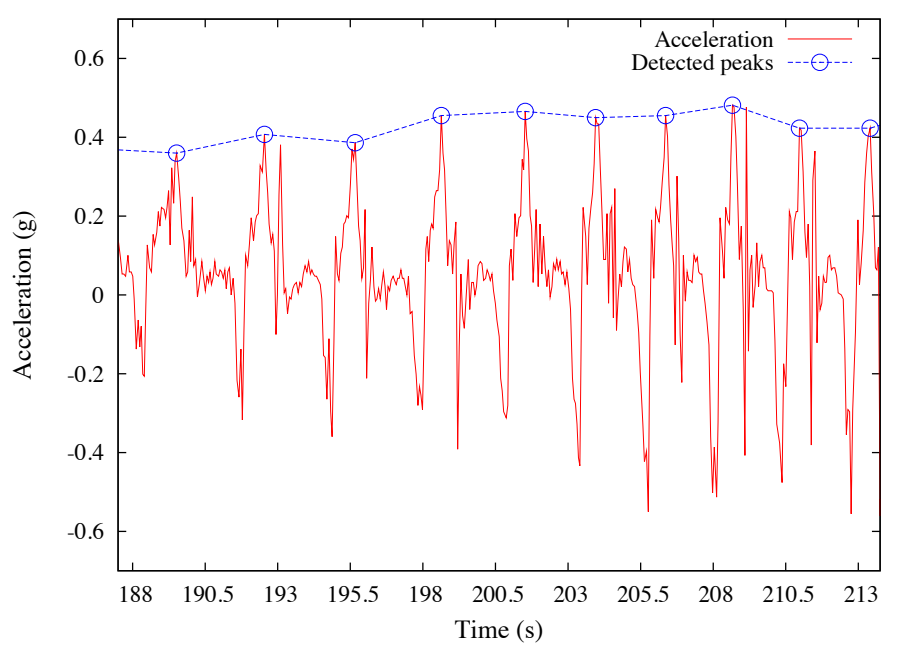

Fig. 3 A peak detection algorithm is applied to acceleration data in order to compute the instantaneous stroke rate, varying here between 19 and 26 strokes per minute. The circle markers show the detected peaks.

fication systems, with help from sensors similar to those presented by Sturm et al. [27] for use in kayak.

\subsection{Sonification design}

\subsubsection{Interactivity}

The main objective of the auditory display is that the rower will learn how to reproduce the movements corresponding to a "good stroke" as assessed either by the coach or by the athlete himself, e.g. through usual haptic perception. It is therefore very important to ensure interactivity so that he will be able to hear in realtime the effects of his own movements and changes in strategy. In this perspective, having a reasonably short latency response is a required in order to maintain the perceptual association within the action-feedback loop.

कo Sonification can also enable a posteriori analysis. Sound computed from logs of training sessions can be generated with an accelerated timestamp in order to divide the time of analysis. This method is commonly used in various domains using auditory display of large sets of data. An illustration is given by Hayward with the audification of seismograms [10]: the analysis of the data, which can cover several hours of recording, can be performed with a time-compression factor of 200. In a similar way, a long training session can be skimmed through rapidly, provided that the listener has received a training beforehand to be able to extract relevant information from the display.

The use of offline listening tests can be arguable when the purpose is to evaluate an interactive sonification system. However, according to Bonebright et al. [2], "when selecting auditory stimuli for use in data sonification applications, active use experiments along with discrimination and identification tests are critical". Whereas active use experiments requires the system to be used in realistic conditions - and therefore in an interactive setting for an interactive sonification system, the two other test categories correspond to an assessment of the perception of auditory stimuli which is usually done in offline conditions. An example of identification test is given by Fernström et al. [6]: a large collection of everyday sounds were assessed for identification, the results giving an idea of the potential success of given stimuli when used as metaphor sounds in an auditory display. Another type of offline evaluation was performed by Walker in [31]: sonification mappings (associations between sound attributes and data dimensions) were assessed through conceptual magnitude estimation, providing ideas about consistency, polarity and perceptual scale inherent to the sonification mappings. Online testing is often limited to after-use surveys and verbal protocols (e.g. "think aloud") due to experimental constraints. Offline listening tests offer more flexibility by enabling the subjects to give quantitative ratings while listening to the sound stimuli at the same time - which would be impossible in the case of elite rowing - and can provide a good insight of the quality of the chosen sound design as it is evaluated alone. On the other hand, the link between perception and action is lost, and there is therefore no immersion of the subject in the system. Nevertheless, for the reasons mentioned above, we consider offline listening tests as complementary to real-time interactive testing. 


\subsubsection{Function and esthetics: a dyadic relationship}

As sonification methods grow more diverse and sophisticated, allowing for a wide range of applications, esthetics of sonification systems has become a specific matter of concern. Introducing the Ars InformaticaArs Musica Esthetic Perspective Space, Vickers and Hogg [28] proposed a classification assigning an esthetic value to auditory displays in the same continuum as musical works. This esthetic value is a major issue in the design of auditory display, especially when the system is supposed to work during long periods.

According to Hansson [9], there exist several philosophical theories concerning the relationship between function and esthetics. On the one hand, the reduction thesis states that the esthetic value of an object is completely determined by its practical function. An expression of this thesis is architectural functionalism, a school of thought claiming that the design of a building should exclusively follow its function. At the other end of the spectrum, the independence thesis considers the two dimensions as entirely independent of each other. Hansson demonstrates that these two extreme theories are untenable and supports an intermediate view: the contributory thesis, which states that esthetics and function are correlated: esthetic judgements are related to perceived functionality to some extent, but not exclusively. Following this view, we can expect users of any sonification system to relate perceived functionality - i.e. the quantity of information they assume to be able to extract from the sound display - to esthetic judgement - i.e. the degree of pleasantness in their experience of the sound, the strength of the correlation being most probably dependent on the context of user tasks (e.g. competitive training vs. recreational activities).

One needs to bear in mind that an auditory display is often much more intrusive than a visualization system. If it turns out to be annoying, the design would be considered as not usable and it will be abandoned quickly. Furthermore, in our particular case, the practice of a sport at a high level is very demanding and an intrusive display would certainly not be welcome. Poor esthetics becomes particularly problematic when the sound feedback is displayed continuously. However, considering the type of information that we want to provide to the rower, we believe that a discrete feedback would not be sufficient. Besides, the unavailability of a biomechanical reference curve makes it difficult to design a display in the form of a warning, that would only be active in case of a digression from this reference. The four models presented in this article were therefore designed as continuous feedback from kinematic quan- tities. Following this strategy, we are aware that achieving a satisfying esthetic quality will be challenging. Furthermore, one should not lose track of the primary goal of sonification, which is to provide useful information to the subject. Improving esthetics might be important with respect to usability, it should not be accomplished at the cost of quality of information communication. From this perspective, the first model Pure tone is expected to be strongly rejected by the rowers. Henkelmann [11], calling such a model "the 'Hello World' sonification", mentioned difficulties regarding esthetics during his experiments, whereas results from Schaffert, Barrass, et al. [1,21-25], indicated that this sonification was fairly popular among the users. Halpern et al. [8] showed that the degree of unpleasantness of a pure tone is of the same order than the sound produced by a pencil sharpener, white noise, and compressed air. Those sounds were rated as less unpleasant than e.g. scraping wood, scraping metal rubbing two pieces of styrofoam together, and scraping slate, but more unpleasant than ecological sounds such as a rotating bicycle tire, jingling keys, and running water. However, a pure tone with gliding frequency is probably the most straightforward display to implement and the most simple one to understand. The real challenge in the design of further models is to preserve this simplicity while improving the rowers' auditory comfort.

\subsubsection{Sonification methods}

Hermann proposed a taxonomy for sonification [12], enumerating the different types of existing sonification methods: Audification, Auditory Icons, Earcons, ParameterMapping Sonification and Model-Based Sonification. Referring to his work, we chose to use the ParameterMapping Sonification method for the quantities for which a continuous feedback was required, i.e. boat acceleration and velocity. In the second sonification model presented below (Musical instruments), additional Earcons are added up in order to give a feedback concerning the current time-lag with respect to a target stroke rate chosen at the beginning of the training. An Earcon is a short sound pattern used to represent a specific event. Detailed descriptions of all the above-mentioned methods are available in the literature (see [32] for a comprehensive classification of sonification methods).

\subsubsection{Models for sonification}

In the next paragraphs, we introduce four sonification models using synthesized sounds to provide a real-time feedback of some kinematic quantities related to the motion of a rowing boat. Data processing is performed 
by a Python script in all the cases. Sound synthesis is done with Python in the first model, Symbian C++ in the second model and Pure Data in the third and fourth models.

\section{Pure tone}

The sound material used in this first attempt to test the principle of sonification on kinematic data characterizing the motion of a rowing boat was a pure tone with gliding frequency. The sonified quantity is the boat velocity, computed as explained in Section 3.1. The tone frequency is coupled to the data using the following mapping:

$f(t)=\alpha \exp [\beta v(t)]$

where $v$ is the velocity integrated from the acceleration data and $\alpha$ and $\beta$ are positive parameters kept constant throughout the experiment, used to keep the frequency band within an audible range. The exponential mapping function follows the representation of pitch in the human auditory system, which is proportional to the logarithm of frequency.

Using such a mapping, the frequency range is not explicitly defined because the extreme values of the velocity for a given data sample cannot be known prior to the experiment. However, the mapping can be entirely defined by assigning a frequency to two reference values of the velocity. For the present experiment, we chose the following reference velocities: $v_{1}=2.5 \mathrm{~m} . \mathrm{s}^{-1}$ and $v_{2}=6 \mathrm{~m} . \mathrm{s}^{-1}$, the interval $\left[v_{1}, v_{2}\right]$ encompassing the velocity range for a single sculler in most of the cases, to which we assigned respectively $f_{1}=35 \mathrm{~Hz}$ and $f_{2}=7000 \mathrm{~Hz}$ in order to avoid unpleasant very high-pitched sounds. The great majority of input velocity data used to create the sound stimuli were actually comprised between $3 \mathrm{~m} . \mathrm{s}^{-1}$ and $5 \mathrm{~m} . \mathrm{s}^{-1}$ (as illustrated in Fig. 2), leading to a resulting frequency varying from $73 \mathrm{~Hz}$ to $1547 \mathrm{~Hz}$, i.e. $D_{2}$ to $G_{6}$ in scientific pitch notation.

This model is similar to sinification implemented and evaluated by Schaffert, Barrass, et al., who mapped acceleration to pitch instead of velocity to pitch in our case.

\section{Musical instruments}

The second sonification system makes use of the MIDI synthesizer built in the mobile phone to generate musical sounds. This has several advantages: polyphonic capabilities allow for the existing data sets to be associated with different instruments, musical sounds are much more friendly to the human ear than sinusoidal tones and having a controller directly incorporated into the device in charge of the data acquisition saves computational resources and time associated to data transfer. The pattern of the generated sound is a "trill" of constant bandwidth - hence not a musical trill strictly speaking - played by pizzicato strings, also using Equation 1 to determine the pitch range of its center frequency. We used exactly the same mapping than in the Pure tone model, apart from the fact that it was clipped so that the resulting frequency would be bounded by the values corresponding to extreme MIDI note numbers $(20 \mathrm{~Hz}$ and $12544 \mathrm{~Hz}$ ), but these limits were never reached in practice. In order to accentuate the expressivity of the trill and to reinforce the perception of a greater speed for a higher pitch, the intertone duration is determined by a hyperbolic tangent-shaped function yielding values between $20 \mathrm{~ms}$ and $220 \mathrm{~ms}$.

Peaks of acceleration detected by the algorithm mentioned in Section 3.1 are used to determine and render the time-lag of the current stroke with respect to the intended stroke rate, chosen by the athlete at the beginning of the training. As shown in Fig. 4, we use the sound of two percussive instruments for providing this information to the rower in the form of an Earcon: the sound of a drum hit is played at once when a peak is detected by the algorithm, then the sound of a ringing bell comes after a constant time delay $\Delta t$ corresponding to the period of the rowing cycle for the chosen stroke rate. The objective for the rower is to synchronize the sound of the bell with the next drum hit. The choice of percussive instruments was motivated by the natural ability for humans to follow rhythmical patterns displayed in the auditory modality in synchronization tasks, as pointed out by Repp and Penel [20].

The idea of using direct Parameter-Mapping Sonification by coupling input data to the pitch of instrumental sounds was also used in xylophone MIDI-fication by Schertenleib, Schaffert, and Barrass, cited in [22] but not retained for the evaluation conducted in [1]. However, this algorithm was a straightforward transposition of sinification and mapped acceleration to pitch, whereas our model maps velocity to the center frequency of a trill. Several other models implemented the sonification of turning points through discrete sound events, but only our model includes a model-driven feedback mechanism.

\section{Wind}

This model, as well as the next one - Car engine, were implemented starting from Pure Data patches available for download on Andy Farnell's personal webpage [5], offering many examples of environmental sound design. This was motivated partly by the assumption that complex sounds are less boring to listen to during long peri- 


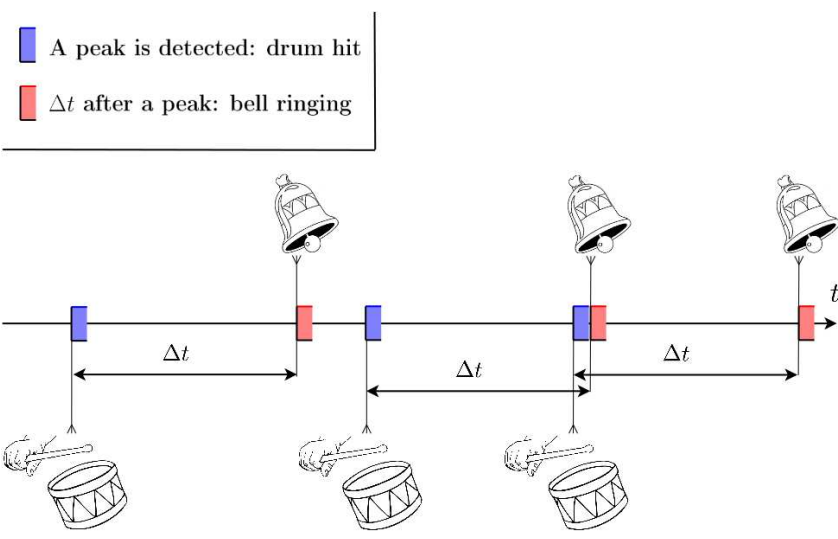

Fig. 4 The model Musical instruments incorporates a model-driven feedback mechanism: following the choice of a specific target stroke rate for the training session, the rower can hear directly if the instantaneous stroke rate deviates from this objective. When a peak is detected in the acceleration, a drum hit is displayed immediately, and a bell rings after a time delay $\Delta t$ that depends on the target stroke rate. The aim is to synchronize the bell with the next drum hit.

ods of time than simple ones, and partly to take advantage of the ecological approach to auditory perception by using environmental sounds to trigger natural associations in the athlete's mind.

This model uses the following metaphorical association: a subject moving with a given velocity with respect to the world would experience the sound of wind, the loudness of which depends on this velocity. Here we use the velocity of the boat as an control parameter to a Pure Data patch generating a synthesized wind sound. The input parameter of this model controls the sound pressure level of the output sound wave. A simple linear scaling of the boat velocity $v$ is performed so that the input parameter stays in the interval $[0,1]$. The synthesized wind sound is directly multiplied by this parameter, which acts as a damping factor.

The sound design is similar to the model weather metaphor assessed in [1], apart from the fact that the mapping associates loudness to velocity whereas Barrass et al. associated both loudness and brightness to acceleration.

\section{Car engine}

In this model we use the metaphor of the car engine: when driving a vehicle, pushing the gas pedal increases the number of revolutions per minute (RPM) of the engine, leading to a characteristic timbre change in the resulting sound, most notably due to a shift of the spectral centroid. Like the previous one, this model was implemented with help from a Pure Data patch created by Farnell [5], including a control parameter for the RPM value. The RPM value is in fact directly related to the angular velocity of the wheels and therefore

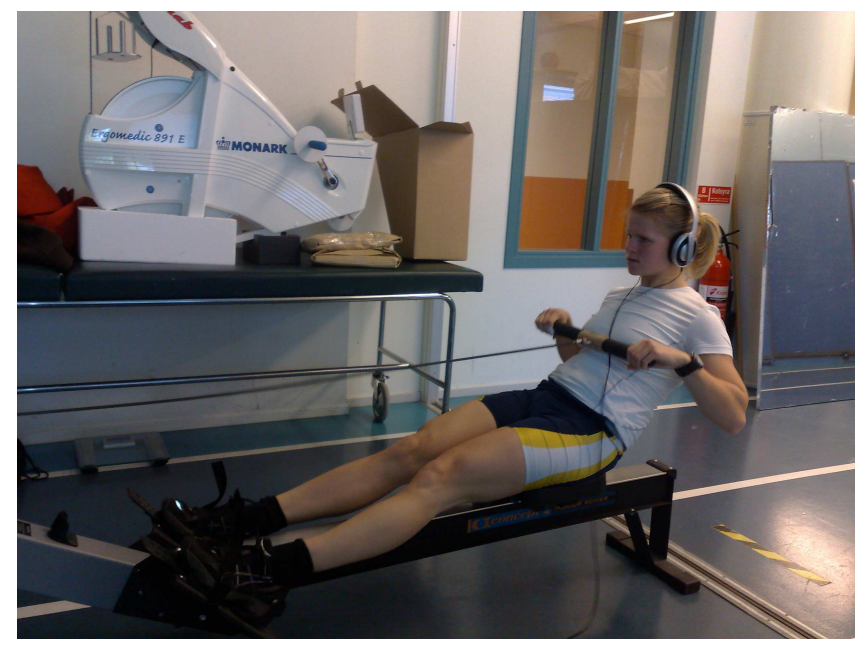

Fig. 5 An informal presentation of the interactive version of the model Musical instruments was made to rowers of the Swedish national team. Acceleration of the sliding seat of an ergometer was used instead of boat acceleration.

to the velocity of a motor vehicle - gear change aside. However, the main idea of our metaphor is not to emulate car driving, but rather to couple the motive force applied by the rower to the oars and foot-stretchers to the motive force applied by the driver to the gas pedal. According to Newton's second law of motion, the motive force produced by the rower is proportionnal to the boat acceleration. Thus, in order to follow the metaphor, the boat acceleration in the direction of propulsion was used as a control parameter. In a same manner as for the previous example, this variable is linearly scaled to an input parameter in the interval $[0,1]$.

\section{Experiment: evaluation of the sonification models}

The objective of the present work is to enhance the training of the rowers, and therefore a complete evaluation of a model should obviously include some interactive testing, i.e. on-water experiments. However, we are still in the process of designing sound models at this stage. The first experiments were intended to collect data as introduced in Section 3.1 in order to perform realistic simulations during the design process.

Only the Musical instruments model was working in real-time at the time of the data collection. It was informally presented to the rowers (Cf. Fig. 5). The interaction was slightly different than in a real rowing situation as the accelerometer was fixed under the sliding seat of an ergometer, yet it was useful in order to outline the interactivity of the system and to verify that the peak detection algorithm was working correctly. 


\subsection{Methodology}

To evaluate and compare the four models, two listening tests were conducted. The first experiment included only two models and served as a pilot study. It was performed at the Bosön sport technology center in Lidingö, Sweden with rowers from the Swedish national team. The second experiment was conducted as an online survey in order to collect answers from a larger panel of athletes. It included the four sonification models. Questionnaires were set up in order to assess the extent of information transmitted by the sound in offline conditions, as well as the preferences of the participants with respect to both function and esthetics.

\subsubsection{Original assumptions}

The questionnaires were designed to investigate the following hypotheses:

a. Since the only input data are acceleration time series, the sonification models allow to differentiate simple characteristics of the data (e.g.: strong, fast) but not to extract more advanced information (e.g.: rower's gender and experience).

b. The sonification models enable a correct estimation of the stroke rate.

c. A strong correlation is expected within two groups of questions corresponding to the same dimension of judgement (following the question labels specified in Section 4.1.3: B1-B4 correspond to function, B5-B7 to esthetics). As explained in Section 3.2.2, the rowers associate the amount of information they assume to be able to extract to the esthetic value given to the sonification model. A weaker but significant correlation is therefore expected between the two subgroups of questions.

d. Due to the personal nature of sound experience, individual preferences will vary strongly from one subject to another. It will nevertheless be interesting to investigate whether an esthetic ranking can be established from the participants' answers.

e. Since our models are far from the stage of a commercial product from a HCI perspective, particularly with respect to usability, a relatively high rejection rate is expected, especially in the case of the Pure tone model which was designed regardless of any esthetic considerations.

\subsubsection{First experiment}

The first listening test included six sound stimuli corresponding to three different data sets sonified by the first two models (Pure tone and Musical instruments).
Table 1 Acceleration samples used in the first experiment: rower information and stroke rate.

\begin{tabular}{lllc}
\hline ID & Level & Gender & Strokes $/$ min \\
\hline 1 & Beginner & Male & 18 \\
2 & International & Male & 26 \\
3 & International & Female & 26 \\
\hline
\end{tabular}

Table 2 Questions for the first experiment: characteristics for each sound stimulus.

\begin{tabular}{lll}
\hline \multicolumn{3}{c}{ "How does it sound?" } \\
A1: & Very weak rowing & Very strong rowing \\
A2: & Very slow rowing & Very fast rowing \\
A3: & Masculine rowing $\leftrightarrow$ & Feminine rowing \\
A4: & Junior rowing & Senior rowing \\
A5: & Not my technique & My technique \\
A6: & Estimate the stroke rate \\
\hline
\end{tabular}

Table 3 Questions for the first experiment: individual preferences concerning the sonification models.

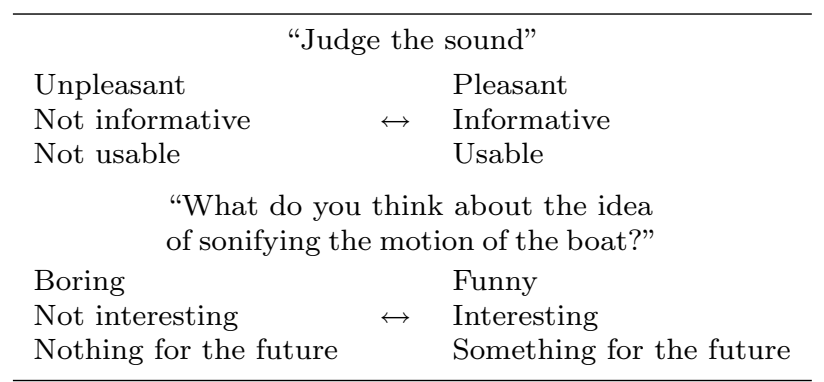

The original acceleration samples are described in Table 1. All sound stimuli were presented randomly. For each stimulus, the participants were asked to judge how they would characterize the sound with respect to given attributes. The questions, referred to as Questions A1A5, were in the form of eleven-step Likert scales with opposite qualities at the two extremities. The list of qualities is given in Table 2 . The participants were then asked to estimate the stroke rate corresponding to each sound stimulus in strokes per minute (Question A6). At the end of the experiment, the participants answered a few questions about esthetics of the models and could give their opinion about the principle of sonification. These questions were also in the form of eleven-step Likert scales, and are specified in Table 3. The participants were finally asked, in the form of a polar question, if they would agree to use this kind of sound during their training.

\subsubsection{Second experiment}

The same structure was used in the second experiment as in the first one. This time, five acceleration samples, 
Table 4 Acceleration samples used in the second experiment: rower information and stroke rate.

\begin{tabular}{lllc}
\hline ID & Level & Gender & Strokes $/$ min \\
\hline 1 & International & Female & 18 \\
2 & Beginner & Male & 18 \\
3 & International & Male & 26 \\
4 & International & Female & 17 \\
5 & International & Female & 26 \\
\hline
\end{tabular}

Table 5 Questions for the second experiment: individual preferences concerning the sonification models.

\begin{tabular}{|c|c|c|c|}
\hline \multicolumn{4}{|c|}{ "How easy was it to understand the sonification?" } \\
\hline B1: & Very difficult & $\leftrightarrow$ & Very easy \\
\hline \multicolumn{4}{|c|}{ "How much information are you able to extract?" } \\
\hline B2: & Very little & $\leftrightarrow$ & Very much \\
\hline \multicolumn{4}{|c|}{ "How much do you recognize the action of the rower? } \\
\hline B3: & Very little & $\leftrightarrow$ & Very much \\
\hline \multicolumn{4}{|c|}{$\begin{array}{l}\text { "To which extent are you able to recognize } \\
\text { characteristic subpatterns of the rowing cycle?" }\end{array}$} \\
\hline B4: & Very little & $\leftrightarrow$ & Very much \\
\hline \multicolumn{4}{|c|}{ "Judge the sound" } \\
\hline B5: & Unpleasant & & Pleasant \\
\hline B6: & Tiring & $\leftrightarrow$ & Relaxing \\
\hline B7: & Intrusive & & Not intrusive \\
\hline
\end{tabular}

described in Table 4, were sonified using the four sonification models. The models were presented randomly and for each model, the five sound stimuli were also randomized. Personal information about rowing experience and musical experience were first collected in order to set up the profile of the participant. Then, for each sound stimulus, the questions were the same as in Table 2 with the exception of the last one (Question A5) which was omitted. Participants were asked to estimate the corresponding stroke rate in strokes per minute (Question A6). Individual preferences concerning the four different sonification models were assessed using the questions presented in Table 5, still using eleven-step Likert scales, which were asked for each model after having evaluated the corresponding five sound stimuli.

The participants were then asked if they would agree to use this sound during their training on water. They could also give their opinion about the project (Cf. Table 3, "What do you think about the idea of sonifying the motion of the boat?"). Finally, they were asked to establish an explicit ranking of the models according to their overall preference.

\subsection{Experimental results}

\subsubsection{Participants}

A total of 7 rowers (2 male, 5 female; mean age: 21.4 years; average rowing experience: 7.4 years), all of international level, took part in the first experiment. A total of 10 rowers of international level ( 6 male, 4 female; mean age: 37.2 years; average rowing experience: 20.1 years) and 13 casual rowers (11 male, 2 female; mean age: 34.4 years; average rowing experience: 9.4 years), took part in the second experiment. Owing to the length of the experiment (approximately 30 minutes), its demanding character and the lack of control over the participants in the context of an online survey, it was expected that some of the participants would not complete the entire experiment. Therefore, the questionnaire was designed to enable the inclusion of partial answers: it was divided into four sections having an identical structure and differing only with respect to the type of auditory stimuli. Partial answers that included complete evaluations of a given sonification model (i.e. the evaluation of the five acceleration samples and the qualitative feedback for the sonification model) were included in the study. The order in which the different sections were successively presented to the subjects was randomized, helping to maintain an even distribution of answers (Pure tone: 20, Musical instruments: 17, Wind: 20, Car engine: 17). Data from parts of the questionnaire that had not been completed were not included in the study. A total of 16 participants completed the whole survey, whereas 7 gave up after having completed the evaluation of at least one sonification model. The statistical analysis was conducted for the entire population of participants as well as for the two subsets (elite rowers, casual rowers). Few significant differences were found in the case of population subsets due to the relatively small number of participants. Moreover, the trends found in this case were confirmed in the analysis including the entire population presented in the next two subsections.

\subsubsection{Characteristics of the sound stimuli}

For both experiments, a two-way ANOVA, repeated measures, with the factors sonification model and acceleration sample was conducted on the participants' values separately for each of the questions related to attributes derived from the sound, as well as for the stroke rate estimate (Questions A1-A6). Pairwise comparisons were analyzed in order to find significant differences for the means of both factors (Bonferroni post hoc comparison, $p<.05$ ). 
Table 6 Estimated stroke rate in the first experiment: average and standard deviation (std.). All values are in strokes per minute.

\begin{tabular}{cccc}
\hline ID & Actual stroke rate & \multicolumn{2}{c}{ Estimated stroke rate: } \\
& & average & std. \\
\hline 1 & 18 & 19.86 & 0.822 \\
2 & 26 & 23.50 & 1.323 \\
3 & 26 & 24.14 & 0.605 \\
\hline
\end{tabular}

Table 7 Estimated stroke rate in the second experiment: average and standard deviation (std.). All values are in strokes per minute.

\begin{tabular}{cccc}
\hline ID & Actual stroke rate & \multicolumn{2}{c}{ Estimated stroke rate: } \\
& & average & std. \\
\hline 1 & 18 & 21.80 & 0.921 \\
2 & 18 & 21.00 & 0.780 \\
3 & 26 & 25.27 & 1.107 \\
4 & 17 & 21.47 & 0.553 \\
5 & 26 & 25.73 & 0.921 \\
\hline
\end{tabular}

In the first experiment, no significant differences were found between the two models. Sample 1 was judged significantly slower than the two others, both with the Likert scale (Question A1) and with the stroke rate estimation summarized in Table 6 (Question A6). Sample 3 was judged significantly stronger than the others (Question A2) and was also judged as closer to the own rowing technique of the participants. No significant difference was found for Questions A3 and A4. The computation of eta-squared showed a large effect size for Questions $\mathrm{A} 1\left(\eta^{2}=.525\right), \mathrm{A} 2\left(\eta^{2}=.370\right), \mathrm{A} 5\left(\eta^{2}=.296\right)$ and $\mathrm{A} 6$ $\left(\eta^{2}=.400\right)$.

In the second experiment, the following significant differences were found between the sonification models: the Car engine model was judged to sound more "masculine" than the Pure tone model (Question A3), the Wind model was judged to sound more "senior" than the models Pure tone and Musical instruments (Question A4). The computation of eta-squared showed a medium effect size for Question A3 $\left(\eta^{2}=.061\right)$ and a small effect size for Question A4 $\left(\eta^{2}=.045\right)$. These significant differences are listed in Table 8 . The following significant differences were found between the acceleration samples: Sample 2 was judged weaker than Sample 5 (Question A1) and slower than Samples 3 and 5 (Question A2). Sample 4 was judged slower than Sample 5. No significant difference was found for Questions $\mathrm{A} 3$ and A4. The value of the stroke rate estimate was significantly different between Sample 3 and Samples 2 and 4, as well as between Sample 5 and Samples 1, 2 and 4 (Question A6). Stroke rate estimates are presented in Table 7 . The computation of eta-squared showed a large effect size for Questions A1 $\left(\eta^{2}=.102\right), \mathrm{A} 2\left(\eta^{2}=.202\right)$ and A6 $\left(\eta^{2}=.250\right)$. These significant differences are listed in Table 8.

\subsubsection{Individual preferences concerning the sonification models}

No comparison was done after the first experiment since the questions about preferences were asked simultaneously for both models. When asked it they would agree to use such a display during training, $57.1 \%$ of the participants answered in the affirmative.

In the second experiment, a two-way ANOVA, repeated measures, with the factor sonification model was conducted on the participants' values separately for each of the questions related to individual preferences (B1-B7). Pairwise comparisons were analyzed in order to find significant differences for the mean of this factor (Bonferroni post hoc comparison, $p<.05$ ). The results show a significant difference for the model Wind with both models Pure tone and Car engine when asking if the sound was Unpleasant/Pleasant (Question B5) and Tiring/Relaxing (Question B6). In both cases, the former was preferred to the two latter, i.e. the Wind sonification model was perceived as more pleasant and relaxing. No significant differences between the sonification models were found for the other questions. The computation of eta-squared showed a large effect size for Questions B5 $\left(\eta^{2}=.404\right)$ and B6 $\left(\eta^{2}=.340\right)$. These significant differences are listed in Table 8. Interestingly, the model Pure tone got the worst mean score for all questions related to functionality (B1-B4). Furthermore, the ranking of models with respect to mean scores was the same for all three questions related to esthetic qualities (B5-B7): the most prefered one was Wind followed by Musical instruments, Pure tone, and Car engine.

The proportion of participants answering that they would use the models for training is shown in Table 9 . The most prefered model in this regard was Wind, and the least prefered one was Pure tone. The table reveals a comparable overall acceptance rate of the sonification models in the two subcategories of subjects (elite rowers, casual rowers). Nevertheless, a detailed analysis of the particular acceptance rate for each sonification model shows noticeable differences between the two groups: casual rowers seemed to prefer the models Pure tone and Musical instruments, whereas elite rowers had a higher acceptance rate for the other models (Wind, Car engine).

The average result given by the subjects' explicit ranking of the sonification models was the following: 1. Wind, 2. Musical instruments, 3. Car engine, 4. Pure tone. 
Table 8 Summary of significant differences found in the second experiment. The function $s r$ represents the stroke rate estimate for each acceleration sample.

\begin{tabular}{|c|c|c|}
\hline Question & Factor: acceleration sample & Factor: sonification model \\
\hline A1 & Sample 2 "weaker" than Sample 5 & none \\
\hline $\mathrm{A} 2$ & Sample 2 "slower" than Sample 3 & none \\
\hline & Sample 2 "slower" than Sample 5 & \\
\hline & Sample 4 "slower" than Sample 5 & \\
\hline A3 & none & Car engine more "masculine" than Pure tone \\
\hline A4 & none & Wind more "senior" than Pure tone \\
\hline & & Wind more "senior" than Musical instruments \\
\hline A6 & $s r($ Sample 1$)<s r($ Sample 5$)$ & none \\
\hline & $s r($ Sample 2$)<\operatorname{sr}($ Sample 3$)$ & \\
\hline & $s r($ Sample 2$)<\operatorname{sr}($ Sample 5) & \\
\hline & $\operatorname{sr}($ Sample 4$)<\operatorname{sr}($ Sample 3$)$ & \\
\hline & $\operatorname{sr}($ Sample 4$)<\operatorname{sr}($ Sample 5$)$ & \\
\hline B1 & \multirow{8}{*}{ not applicable } & none \\
\hline $\mathrm{B} 2$ & & none \\
\hline B3 & & none \\
\hline B4 & & none \\
\hline B5 & & Wind more "pleasant" than Pure tone \\
\hline \multirow{3}{*}{ B6 } & & Wind more "pleasant" than Car engine \\
\hline & & Wind more "relaxing" than Pure tone \\
\hline & & Wind more "relaxing" than Car engine \\
\hline
\end{tabular}

Table 9 Proportion of rowers stating that they would use the model during their training on water.

\begin{tabular}{llll}
\hline \multicolumn{4}{c}{ Positive answers } \\
& $\begin{array}{c}\text { Casual } \\
\text { rowers }\end{array}$ & $\begin{array}{l}\text { Elite } \\
\text { rowers }\end{array}$ & $\begin{array}{l}\text { All } \\
\text { rowers }\end{array}$ \\
\hline Pure tone & 30.0 & 20.0 & 25.0 \\
Musical instruments & 44.4 & 12.5 & 29.4 \\
Wind & 27.3 & 55.6 & 40.0 \\
Car engine & 22.2 & 37.5 & 29.4 \\
All models & 30.8 & 29.7 & 30.3 \\
\hline
\end{tabular}

Finally, bivariate correlations between the answers to questions related to individual preferences were analyzed by computing the Pearson product-moment correlation coefficient. The considered variables were the answers by all participants to the questions B1-B7 for all sound models $(N=74)$. The resulting correlation matrix is shown in Table 10. Significant correlations $(p<.01)$ were found for almost all pairs of questions: only B7 (judging the intrusive character of the model) was not significantly correlated with B1 and B2, and was correlated to the .05 level with B3. Furthermore, the strongest correlations $(r>.500)$ were found for answers belonging to each of the two predicted clusters (B1-B4 and B5-B7).

\section{Discussion}

Questions about characteristics of the sound stimuli were asked in order to assess the ability of the participants to extract information from the sonification mod-
Table 10 Correlation matrix for answers to questions relative to individual preferences (B1-B7), $N=74$. Gray-colored cells show the predicted correlation clusters.

\begin{tabular}{|c|c|c|c|c|c|c|c|c|}
\hline & & B1 & B2 & B3 & B4 & B5 & B6 & B7 \\
\hline B1 & $\begin{array}{l}\text { Pearson Correlation } \\
\text { Sig. (2-tailed) }\end{array}$ & 1 & & & & & & \\
\hline B2 & $\begin{array}{l}\text { Pearson Correlation } \\
\text { Sig. (2-tailed) }\end{array}$ & $\begin{array}{r}.786^{2 \pi} \\
.000\end{array}$ & 1 & & & & & \\
\hline B3 & $\begin{array}{l}\text { Pearson Correlation } \\
\text { Sig. (2-tailed) }\end{array}$ & $\begin{array}{l}.760^{* 2} \\
.000\end{array}$ & $\begin{array}{r}.881^{2 \pi} \\
.000\end{array}$ & 1 & & & & \\
\hline B4 & $\begin{array}{l}\text { Pearson Correlation } \\
\text { Sig. (2-tailed) }\end{array}$ & $\begin{array}{l}.677^{27} \\
.000\end{array}$ & $\begin{array}{c}.796^{22} \\
.000\end{array}$ & $\begin{array}{r}.827^{27} \\
.000\end{array}$ & 1 & & & \\
\hline B5 & $\begin{array}{l}\text { Pearson Correlation } \\
\text { Sig. (2-tailed) }\end{array}$ & $\begin{array}{r}.363^{27} \\
.001\end{array}$ & $\begin{array}{r}.421^{2 *} \\
.000\end{array}$ & $\begin{array}{r}.418^{27} \\
.000\end{array}$ & $\begin{array}{r}.492^{* 2} \\
.000\end{array}$ & 1 & & \\
\hline B6 & $\begin{array}{l}\text { Pearson Correlation } \\
\text { Sig. (2-tailed) }\end{array}$ & $\begin{array}{r}.326^{* 2} \\
.005\end{array}$ & $\begin{array}{r}.391^{27} \\
.001\end{array}$ & $\begin{array}{r}.368^{2 *} \\
.001\end{array}$ & $\begin{array}{r}.440^{2 *} \\
.000\end{array}$ & $\begin{array}{l}.851^{2 *} \\
.000\end{array}$ & 1 & \\
\hline B7 & $\begin{array}{l}\text { Pearson Correlation } \\
\text { Sig. (2-tailed) }\end{array}$ & $\begin{array}{l}.160 \\
.173\end{array}$ & $\begin{array}{l}.227 \\
.052\end{array}$ & $\begin{array}{l}.258^{2} \\
.026\end{array}$ & $\begin{array}{r}.322^{22} \\
.005\end{array}$ & $\begin{array}{r}.598^{22} \\
.000\end{array}$ & $\begin{array}{r}.626^{25} \\
.000\end{array}$ & 1 \\
\hline
\end{tabular}

els. One acceleration sample corresponded to a beginner rower and all the others to athletes from the Swedish national team. In both experiments, the participants succeeded very well to spot the sample of the beginner, assessing the resulting sounds as corresponding to a weaker and slower rowing technique. However, the subjects failed to associate this information to the inexperience of that particular rower. This might indicate that they didn't believe to be able to extract advanced properties such as the gender and the experience of the rower from models being based solely on one kinematic quantity as input parameter: no significant differences were found between the answers to these questions for the factor acceleration sample. The four models led to comparable results in information extraction. The Car engine model was rated as more "masculine" and the 
Wind model was rated as more "senior". However, the effect size was found respectively small and medium for these two significant differences.

Although not matching perfectly the actual numerical values, estimation of the stroke rate revealed a clear separation between the two groups of acceleration samples corresponding to $17-18$ and 26 strokes per minute, as shown in Tables 6 and 7. No significant differences were found between the models, which shows that more complex sounds did not alter the extraction of this particular information by the participants.

Answers to questions concerning individual preferences towards the models were all significantly correlated with each other. According to our expectations, larger correlations were found within two categories (function, esthetics). In addition, significant correlations also appeared between elements of these two subsets. This indicates that rowers associated to some extent the esthetic quality of a model to the amount of information they assumed to be able to extract from it.

Only a few significant differences were found in the qualitative ratings of the models, which underlines the personal character of the experience of sound and therefore illustrates the importance of having various sound models available for the athletes to choose among. However, the analysis of mean scores enabled to observe that Pure tone was considered the least functional model, and to establish a ranking with respect to esthetic ratings: 1. Wind, 2. Musical instruments, 3. Pure tone, 4. Car engine. Explicit ranking and acceptance rate led to similar rankings, with the exception that Pure tone was ranked lower than Car engine in both cases. Altogether, this indicates that the participants ranked the models according to the esthetic value it was awarded rather than according to how informative they thought the model was, although these two properties have been shown to be interrelated. Nevertheless, a presumed poor functionality - as for Pure tone - proved to have a certain influence on the overall acceptance of the system.

As mentioned in Section 3.2.4, two models using a similar sound material as our models were assessed in [1]: sinification and weather metaphor, corresponding respectively to Pure tone and Wind. It is therefore interesting to compare the results obtained by these two pairs of models. In the present work, Wind was clearly the most preferred model, receiving the highest ratings both with respect to esthetic qualities and by overall judgement (explicit ranking and acceptance rate). By contrast, weather metaphor was the least preferred models of [1] in terms of time of use, which was considered as the main criterion for evaluation. Nevertheless, it was shown in post-trial interviews that it was well appreciated, being mentioned as the "most liked" model by more subjects than as the "least liked" one. In the present work, Pure tone was our least popular model, ranking as penultimate with respect to esthetic qualities, and as last with respect to functionality and by overall judgement. By contrast, sinification was the second most preferred model in terms of time of use. Post-trial interviews showed that it was mentioned as the "most liked" and as the "least liked" model by a comparable number of subjects. These discrepancies between the two studies can be explained by several factors: the couplings were not exactly the same (using acceleration as input parameter while we chose to display velocity in the corresponding models), the experimental conditions were very different (interactive testing in the context of recreational sports, while we performed listening tests in the context of competitive training), and so was the user population as well (HCI researchers in [1], elite and casual rowers in our study).

Remarkably, the experimental protocol seemed to strongly influence the overall ratings of the sonification models, the participants showing less leniency in the more impersonal mode of an online survey. This is reflected by the significant difference in the proportion of rowers willing to use the models $(57.1 \%$ in the pilot study, $30.3 \%$ in the second experiment). A possible influential factor could be the fact that rowers of international level are more disposed to accept annoying additions to their training provided that it would improve the performance, but the separation into two subcategories (elite rowers, casual rowers) in the second experiment yields to comparable overall acceptance percentages (Cf. Table 9), indicating clearly that this is not the case. The fact that Schaffert et al. found even higher acceptance rates [22-24] suggests that trying the sonification system in real-time conditions before the evaluation could have a significant influence on the degree of acceptance of the models.

A more detailed analysis of the acceptance rate revealed differences in the model preferences between the two subcategories of subjects (elite rowers, casual rowers). This is consistent with the finding - in a different context, namely recreational sports - by Barrass et al. [1] that there exist general trends in preferences, and "subgroups with different aesthetic and functional requirements". In the present experiment, elite rowers seemed to prefer models making use of metaphorical associations (Wind, Car engine) whereas casual rowers showed a better acceptance of "musical" models ( $\mathrm{Mu}$ sical instruments, Pure tone). An explanation could be that the models using ecological sounds and metaphors lead to a lower cognitive load than others, which is most certainely crucial when applied in the context of a demanding training. The present study is however lim- 
ited by the relatively small number of participants, and therefore only allows for speculation in this regard: further studies would be required in order to draw more definitive conclusions.

Informal feedback from rowers during direct conversations or through free comments in the questionnaires revealed a certain interest for the principle of sonification. Some of them came up with ideas for other potential uses of sound for enhancing the training, e.g. to help the synchronization of a crew. On the other hand, they were particularly critical about esthetics of the sounds. Some stated that they could use one of the models but staunchly rejected all the others. Interestingly, this preferred model was not always the same among the participants. Some pointed out that they could probably not stand a system producing sound continuously but that a discrete feedback as the one used in Musical instruments would be useful. Several rowers suggested to use synthesized water sounds, e.g. splashing and flowing water. Interestingly, this idea was implemented in the ecological sonification model by Williamson and Murray-Smith cited in [22] but not further evaluated, to our knowledge.

\section{Conclusions and future work}

We presented an advanced quantitative evaluation of four different models for the sonification of rowing. The lack of advanced methods for the evaluation of sonification systems is a critical issue for the field of auditory display. Few quantitative approaches enabling an objective comparison of different sonification paradigms have been used in the past, as it was shown by Vogt [29] in a review of evaluation works published within the International Community for Auditory Display (ICAD) between 1992 and 2009.

In the present study, it was revealed that at the current state of development, our sonification models allow to differentiate simple characteristics of the data (strong, fast) but not to extract more advanced information (gender, experience). The listeners were able to fairly estimate the stroke rate of randomized acceleration samples, notwithstanding a bias in the estimation of its absolute value. The estimation of the stroke rate did not depend on the sonification model that was used. Nevertheless, a greater variety of numerical values for the stroke rate should be used in future experiments in order to confirm these results. It was found that rowers correlated the amount of information that they assumed to be able to extract from a particular sonification model to its esthetic appreciation. Despite the personal nature of sound experience, a ranking could be established with respect to both esthetic and overall preferences, the latter being rather influenced by esthetic aspects than by functional aspects. However, marked differences were found in the acceptance rate of specific sonification models between two subcategories (elite rowers and casual rowers), while the overall acceptance rate of the principle of sonification was comparable. Finally, the nature of the experimental protocol was found to have a major influence on ratings and acceptance rate of the sonification models.

Future work should include on-water experiments to assess the interactivity of the system when the sonification works in real-time. The development of further models of advanced complexity appears to be necessary, in order to provide more options of sound display to the rowers, as they experience the sonification models in a very personal way. The existing models could be modified, keeping in mind that the esthetic quality of any sound display should be taken into serious consideration. For example, specific scales could be used in Musical instruments instead of a chromatic ascent. More sensors, for example biofeedback probes, should be used in the data collection so that the athletes would be able to extract more advanced information from the sound. Finally, there is a need for a general reflexion to determine which specific sound parameters can be coupled to particular physical quantities in a relevant manner.

Acknowledgements This work was supported by the Swedish Research Council, Grant Nr. 2010-4654, by the Olympic Performance Center (OPC) SONEA project, and by the EUICT SAME project (FP7-ICT- STREP-215749). The author would like to thank the rowers and trainers who took part in the experiments. Many thanks to the people who helped spreading the online survey among the community of rowers and to the rowers and researchers who answered.

\section{References}

1. Barrass, S., Schaffert, N., Barrass, T.: Probing preferences between six designs of interactive sonifications for recreational sports, health and fitness. In: R. Bresin, T. Hermann, A. Hunt (eds.) Proceedings of ISon 2010, 3rd Interactive Sonification Workshop. KTH, Stockholm, Sweden (2010)

2. Bonebright, T.L., Miner, N.E., Goldsmith, T.E., Caudell, T.P.: Data collection and analysis techniques for evaluating the perceptual qualities of auditory stimuli. ACM Transactions on Applied Perception 2(4), 505-516 (2005)

3. Effenberg, A.O.: Movement sonification: Effects on perception and action. IEEE Multimedia 12(2), 53-59 (2005)

4. Eriksson, M., Bresin, R.: Improving running mechanics by use of interactive sonification. In: R. Bresin, T. Hermann, A. Hunt (eds.) Proceedings of ISon 2010, 3rd Interactive Sonification Workshop. KTH, Stockholm, Sweden (2010) 
5. Farnell, A.: URL http://obiwannabe.co.uk. Personal website. Retrieved December 7, 2011

6. Fernström, M., Brazil, E., Bannon, L.: HCI design and interactive sonification for fingers and ears. IEEE Multimedia 12(2), 36-44 (2005)

7. Godbout, A., Boyd, J.E.: Corrective sonic feedback for speed skating: a case study. In: Proceedings of the 16th International Conference on Auditory Display. Washington, DC, USA (2010)

8. Halpern, D.L., Blake, R., Hillenbrand, J.: Psychoacoustics of a chilling sound. Perception \& Psychophysics 39(2), 77-80 (1986)

9. Hansson, S.O.: Aesthetic functionalism. Contemporary Aesthetics 3, online publication (2005)

10. Hayward, C.: Listening to the Earth sing. In: G. Kramer (ed.) Auditory Display - Sonification, Audification and Auditory Interfaces, SFI studies in the sciences of complexity, pp. 369-404. Addison Wesley Longman (1992)

11. Henkelmann, C.: Improving the aesthetic quality of realtime motion data sonification. Tech. Rep. CG-2007-4, Universität Bonn (2007)

12. Hermann, T.: Taxonomy and definitions for sonification and auditory display. In: P. Susini, O. Warusfel (eds.) Proceedings of the 14th International Conference on $\mathrm{Au}-$ ditory Display. IRCAM, Paris, France (2008)

13. Hermann, T., Höner, O., Ritter, H.J.: AcouMotion - An interactive sonification system for acoustic motion control. In: S. Gibet, N. Courty, J.F. Kamp (eds.) Lecture Notes in Computer Science, vol. 3881, pp. 312-323. Springer, Berlin, Heidelberg (2006)

14. Hermann, T., Zehe, S.: Sonified aerobics - Interactive sonification of coordinated body movements. In: G. Wersényi, D. Worrall (eds.) Proceedings of the 17th International Conference on Auditory Display. OPAKFI Egyesület, Budapest, Hungary (2011)

15. Hofmijster, M.J.: Mechanics and energetics of rowing. Ph.D. thesis, Vrije Universiteit, Amsterdam (2010)

16. Hummel, J., Hermann, T., Frauenberger, C., Stockman, T.: Interactive sonification of German wheel sports movements. In: R. Bresin, T. Hermann, A. Hunt (eds.) Proceedings of ISon 2010, 3rd Interactive Sonification Workshop. KTH, Stockholm, Sweden (2010)

17. Kleiman-Weiner, M., Berger, J.: The sound of one arm swinging: a model for multidimensional auditory display of physical motion. In: T. Stockman, L.V. Nickerson, C. Frauenberger, A.D.N. Edwards, D. Brock (eds.) Proceedings of the 12th International Conference on Auditory Display. Department of Computer Science, Queen Mary, University of London, London, UK (2006)

18. Kleshnev, V.: Rowing faster, chap. 18: Technology for technique improvement, pp. 209-225. Human Kinetics, Inc. (2005)

19. McBride, M.: Rowing faster, chap. 10: Rowing biomechanics, pp. 111-123. Human Kinetics, Inc. (2005)

20. Repp, B.H., Penel, A.: Rhythmic movement is attracted more strongly to auditory than to visual rhythms. Psychol. Res. 68(4), 252-270 (2004)

21. Schaffert, N., Gehret, R., Effenberg, A.O., Mattes, K.: The sonified boat motion as the characteristic rhythm of several stroke rate steps. In: World Congress of Performance Analysis of Sport VII. Magdeburg, Germany (2008)

22. Schaffert, N., Mattes, K., Barrass, S., Effenberg, A.O.: Exploring function and aesthetics in sonifications for elite sports. In: Proceedings of the 2nd International Conference on Music Communication Science. Sydney, Australia (2009)
23. Schaffert, N., Mattes, K., Effenberg, A.O.: A sound design for the purposes of movement optimisation in elite sport (using the example of rowing). In: M. Aramaki, R. Kronland-Martinet, S. Ystad, K. Jensen (eds.) Proceedings of the 15th International Conference on Auditory Display. Re:New - Digital Arts Forum, Copenhagen, Denmark (2009)

24. Schaffert, N., Mattes, K., Effenberg, A.O.: Listen to the boat motion: acoustic information for elite rowers. In: R. Bresin, T. Hermann, A. Hunt (eds.) Proceedings of ISon 2010, 3rd Interactive Sonification Workshop. KTH, Stockholm, Sweden (2010)

25. Schaffert, N., Mattes, K., Effenberg, A.O.: The sound of rowing stroke cycles as acoustic feedback. In: G. Wersényi, D. Worrall (eds.) Proceedings of the 17th International Conference on Auditory Display. OPAKFI Egyesület, Budapest, Hungary (2011)

26. Soper, C., Hume, P.A.: Towards an ideal rowing technique for performance: The contributions from biomechanics. Sports Medicine 34(12), 825-848(24) (2004)

27. Sturm, D., Yousaf, K., Eriksson, M.: A wireless, unobtrusive kayak sensor network enabling feedback solutions. In: L. O'Conner (ed.) Proceedings of the International Conference on Body Sensor Networks. IEEE Computer Society, Biopolis, Singapore (2010)

28. Vickers, P., Hogg, B.: Sonification abstraite/Sonification concrète: An 'æsthetic persepective space' for classifying auditory displays in the ars musica domain. In: T. Stockman, L.V. Nickerson, C. Frauenberger, A.D.N. Edwards, D. Brock (eds.) Proceedings of the 12th International Conference on Auditory Display. Department of Computer Science, Queen Mary, University of London, London, UK (2006)

29. Vogt, K.: A quantitative approach to sonifications. In: G. Wersényi, D. Worrall (eds.) Proceedings of the 17th International Conference on Auditory Display. OPAKFI Egyesület, Budapest, Hungary (2011)

30. Vogt, K., Pirrò, D., Kobenz, I., Höldrich, R., Eckel, G.: Physiosonic - Movement sonification as auditory feedback. In: M. Aramaki, R. Kronland-Martinet, S. Ystad, K. Jensen (eds.) Proceedings of the 15th International Conference on Auditory Display. Re:New - Digital Arts Forum, Copenhagen, Denmark (2009)

31. Walker, B.N.: Consistency of magnitude estimations with conceptual data dimensions used for sonification. Applied Cognitive Psychology 21, 579-599 (2007)

32. Worrall, D.: Sonification and information: Concepts, instruments and techniques, chap. 2: An overview of sonification. Ph.D. thesis, University of Canberra, Canberra, Australia (2009) 